\title{
Desigualdad educativa en Costa Rica: la brecha entre estudiantes de colegios públicos y privados. Análisis con los resultados de la evaluación internacional PISA
}

Andrés Fernández A. y Roberto Del Valle A.

En este artículo se presentan los principales resultados del Programa Internacional de Evaluación de Estudiantes (PISA) de 2009 para Costa Rica y se analiza la brecha por tipo de colegio, donde se evidencia que los estudiantes que asisten a colegios privados obtienen mejores puntajes que aquellos de colegios públicos. Sin embargo, mediante la estimación de una función de producción educativa (FPE) se demuestra que esta brecha no obedece en su totalidad al tipo de administración del centro educacional al que concurren los estudiantes, sino más bien a los factores familiares y las características personales de los estudiantes, especialmente al año escolar que cursa el joven en el momento de realizar la prueba PISA.

PALABRAS CLAVE

CLASIFICACIÓN JEL
Educación, calidad de la educación, rendimiento escolar, medición, escuelas públicas, escuelas privadas, estadísticas de la educación, Costa Rica

$\mathrm{I} 21, \mathrm{I} 24, \mathrm{O} 38$

Andrés Fernández A. es Licenciado en Economía de la Universidad de Costa Rica. affa17@gmail.com Roberto Del Valle A. es Licenciado en Economía de la Universidad de Costa Rica. roberto.dva@gmail.com 


\section{I}

\section{Introducción}

En el año 2010, Costa Rica participó por primera vez en el Programa Internacional de Evaluación de Estudiantes (PISA, por sus siglas en inglés), bajo el proyecto denominado PISA 2009 PLUS, mediante la aplicación de una prueba a una muestra de jóvenes costarricenses de 15 años de edad de diferentes colegios del país. En diciembre de 2011 fueron publicados los resultados, que ubican a Costa Rica como el segundo mejor país en América Latina en las áreas de lectura y ciencias, y quinto en matemáticas, aunque muy por debajo de los resultados de países miembros de la Organización de Cooperación y Desarrollo Económicos (OCDE).

Más allá de los resultados generales, los datos obtenidos por medio de la evaluación PISA, junto con la información proveniente de los cuestionarios aplicados a estudiantes y directores de colegio, permiten formarse una idea respecto de la calidad del sistema educativo costarricense y brindan la oportunidad de conocer no solo el estado de la educación costarricense con respecto a la de otros países, sino también las diferencias educativas que existen en el propio país.

Si bien es cierto que la brecha en puntuación que separa a Costa Rica del grupo de países desarrollados es amplia y merece un análisis más detallado, el estudio de

$\square$ Los autores agradecen la colaboración en la discusión de los resultados de Ronulfo Jiménez Rodríguez, profesor de Economía de la Universidad de Costa Rica, así como de Pablo Zoido, analista del programa PISA, por la orientación en el manejo de los datos. las brechas educativas dentro de un mismo país puede resultar más relevante a la hora de formular políticas educacionales tendientes a disminuir la desigualdad en la calidad de la educación y ofrecer las mismas oportunidades de instrucción para todos los jóvenes.

El presente artículo tiene como objetivos realizar un análisis detallado del rendimiento de los jóvenes costarricenses en la evaluación PISA del año 2009, de los principales factores que influyen en el desempeño de los estudiantes en dicha evaluación y de las diferencias en el rendimiento educativo que se producen entre los colegios públicos y privados.

Luego de esta Introducción, en la sección II se realiza un breve resumen de la situación actual del sistema educativo costarricense. En la sección III se explica en qué consiste la evaluación PISA y se exponen los resultados obtenidos en Costa Rica. Debido a la novedad de los datos para el país y a la escasa difusión de los resultados derivados de la prueba, en la sección IV se efectúa una comparación de los resultados generales de Costa Rica con los de otros países de la región.

En la sección $\mathrm{V}$ se construye una función de producción educativa para analizar los determinantes del rendimiento de los jóvenes en la prueba PISA. En la sección VI se exponen los resultados a nivel nacional. Posteriormente, se utiliza la técnica de descomposición de Fields para analizar los determinantes en las diferencias que se producen en el rendimiento de los estudiantes. Por último, en la sección VII se entregan las conclusiones del estudio. 


\section{II}

\section{La educación en Costa Rica}

\section{El sistema educativo costarricense}

En Costa Rica el sistema educativo formal se encuentra dividido en: Educación Pre-escolar, Educación General Básica (EGB), Educación Diversificada y Educación Superior. La Educación Pre-escolar y la Educación General Básica son obligatorias y ambas, junto con la Educación Diversificada, son gratuitas y costeadas por el Estado.

La EGB consta de tres ciclos obligatorios. El $1^{\mathrm{er}}$ ciclo (primero a tercer año) y el $2^{\circ}$ ciclo (cuarto a sexto año) constituyen la educación primaria. Al finalizar el $2^{\circ}$ ciclo el estudiante recibe un Diploma de Conclusión de Enseñanza Primaria. El $3^{\text {er }}$ ciclo (séptimo a noveno año) es el último ciclo obligatorio. La educación secundaria consta del 3er ciclo junto con un $4^{\circ}$ ciclo denominado Educación Diversificada. La Educación Diversificada tiene una duración de 2 a 3 años y se subdivide en tres ramas: la rama académica y la rama artística tienen una duración de 2 años (décimo y undécimo), mientras que la rama técnica (impartida en colegios técnicos) consta de tres años (décimo, undécimo y duodécimo) y se puede enfocar en una de las siguientes modalidades: industrial, comercial o agropecuario. La rama académica finaliza una vez que el estudiante aprueba los exámenes finales de bachillerato, lo que le otorga el título de Bachiller en Educación Media. Los estudiantes de las ramas artística y técnica también pueden optar al título de Bachiller siempre y cuando aprueben los exámenes finales de bachillerato. Además, los estudiantes de la rama técnica obtienen un título de técnico medio una vez concluidos sus estudios ${ }^{1}$.

\footnotetext{
1 Además de la enseñanza tradicional en escuelas y colegios diurnos, existen otras modalidades de estudio que están dirigidas a jóvenes y adultos que por alguna razón no lograron asistir al sistema educativo formal. Dentro de estas modalidades se encuentran las escuelas y colegios nocturnos, así como programas especiales como Primaria y Secundaria por suficiencia, Colegio a Distancia, Aula Abierta, Nuevas Oportunidades y Centro Integrado de Educación para Jóvenes y Adultos (CINDEA)
}

\section{Costa Rica y las evaluaciones internacionales}

La prueba PISA no es la primera evaluación internacional en la que Costa Rica participa. El país centroamericano forma parte del Laboratorio Latinoamericano de Evaluación de la Calidad de la Educación (LLECE) de la Oficina Regional de Educación de la UNESCo para América Latina y el Caribe, en el que se evaluaron estudiantes de $3^{\circ}$ y $4^{\circ}$ grados de educación primaria en el año 1997 y de $3^{\circ}$ y $6^{\circ}$ grados entre los años 2004 y 2008 (Segundo Estudio Regional Comparativo y Explicativo (SERCE)).

En la primera evaluación, Costa Rica no fue considerado en los informes de resultados ni en los parámetros estadísticos de los diversos estudios, debido a que los datos presentados por el país no tuvieron la consistencia técnica requerida y exigida por el LLECE. En la segunda evaluación, Costa Rica demostró ser uno de los mejores de la región (16 países evaluados), ubicando a sus estudiantes de tercer y sexto grado en el tercer lugar en matemáticas y en segundo lugar en lectura (UNESCO, 2008).

\section{Estudios de calidad educativa en Costa Rica}

La primera estimación del efecto de diversos factores en el rendimiento educativo en Costa Rica data de 1980 (Díaz y Jiménez, 1980). Mediante la estimación de una función de producción educativa con datos a nivel cantonal, los autores concluyen que en el rendimiento educativo influyen tanto los insumos escolares como los factores socioeconómicos. Moreira (2009) analiza la influencia de diversos factores en las puntuaciones obtenidas por estudiantes de undécimo año de colegios académicos diurnos en la prueba nacional de bachillerato de matemáticas del año 2004; a través del análisis multinivel, el autor deduce que los factores endógenos como el historial académico del estudiante, y específicamente la condición de repitencia y el nivel educativo de los padres, se relacionan con diferencias en las puntuaciones obtenidas.

Sin embargo, no se dispone de estudios en que se utilicen bases de datos de evaluaciones internacionales para Costa Rica, por lo que esta investigación se convierte en uno de los primeros aportes al debate sobre calidad y desigualdad educativa. 


\section{III \\ El Programa Internacional de Evaluación de Estudiantes (PISA)}

\section{1. ¿En qué consiste la evaluación internacional PISA?}

El Programa Internacional de Evaluación de Estudiantes (PISA) es un esfuerzo conjunto entre los países miembros de la OCDE, cuyo propósito consiste en medir la capacidad de los estudiantes de 15 años de edad — que están por concluir el ciclo de escolaridad obligatoria - para afrontar los retos de la sociedad actual.

La evaluación tiene una visión a futuro: en lugar de centrarse en medir conocimientos específicos de acuerdo con los currículos o planes de estudio de cada centro educativo, se evalúa la capacidad de los estudiantes para utilizar sus conocimientos y habilidades con miras a afrontar los retos de la vida real.

Las encuestas y evaluaciones del programa PISA se realizan cada tres años y aunque en todas se miden habilidades y conocimientos en lectura, ciencias y matemáticas, cada evaluación se focaliza en una de estas tres áreas, dedicando cerca del $60 \%$ de los ítems a la evaluación del área en que se centra la prueba. La primera de estas evaluaciones se aplicó en el año 2000 y tuvo énfasis en lectura; la segunda prueba se aplicó en 2003 y se concentró en matemáticas; la tercera correspondió al año 2006 y en ella se evaluaron habilidades en ciencias. Con estas tres pruebas concluyó el primer ciclo completo de evaluaciones del programa PISA.

El segundo ciclo de pruebas se inició en 2009 y su evaluación puso el acento en la lectura. Esta fue la primera evaluación en la que Costa Rica tomó parte dentro del programa PISA. La última de estas evaluaciones se realizó recientemente en el año 2012.

Además de la medición de habilidades y conocimientos de los estudiantes mediante una prueba escrita, en la evaluación PISA también se aplican cuestionarios a los estudiantes y directores de colegios para recolectar información que permita analizar y contextualizar adecuadamente los resultados de las pruebas.

\section{Los resultados de PISA 2009: ubicación de Costa Rica a nivel mundial}

Originalmente, en el año 2009 participaron 64 países en la evaluación internacional del programa PISA, 9 de los cuales pertenecen a la región latinoamericana. En el año 2010, diez países adicionales participaron dentro del proyecto PISA 2009 PLUS, a los cuales se les aplicó la misma evaluación que a sus contrapartes en el año 2009. El único país latinoamericano que se unió a la evaluación en el proyecto PISA 2009 PLUs fue Costa Rica.

Los valores del rendimiento de los estudiantes en las evaluaciones se denominan Valores Plausibles y son considerados como variables latentes continuas, lo que significa que se tratan como cantidades que son medibles, pero para las que no existe un instrumento de medida ${ }^{2}$, ya que los ítems no son evaluados con puntuaciones específicas, sino que en la evaluación PISA se definen escalas a partir de las cuales se realizan las comparaciones. Así, a fin de facilitar la interpretación de las notas asignadas a los estudiantes, las escalas en lectura, matemáticas y ciencias fueron diseñadas para tener una nota promedio de 500 puntos y una desviación estándar de 100 puntos con respecto a los países miembros de la OCDE. Esto significa que cerca de dos tercios de los estudiantes de países miembros de esta organización obtienen notas entre los 400 y 600 puntos (OCDE, 2009, pág. 136).

En lectura, los países latinoamericanos aparecen en la segunda mitad de la lista, y todos tuvieron rendimientos promedio inferiores a la media de los países miembros de la OCDE (véase el cuadro A.1 del anexo). Chile se ubica en el primer lugar en lectura en la región latinoamericana con 449 puntos, seguido de Costa Rica con 443 puntos.

En la evaluación de matemáticas, el Uruguay se ubica en el primer lugar del área latinoamericana con 427 puntos. Costa Rica, con tan solo 409 puntos, se sitúa en el quinto lugar latinoamericano y en el lugar 55 a nivel general, siendo esta la peor ubicación y el rendimiento más bajo del país en las tres áreas de interés. En ciencias, la situación es similar a la del área de lectura: Chile en el primer lugar con 447 puntos y Costa Rica en el segundo con 430 puntos.

\footnotetext{
2 En este tipo de evaluación educativa, basada en el modelo estadístico Rasch, no se añaden puntos adicionales al estudiante por cada respuesta correcta, sino de acuerdo con el tipo de respuesta dada. Mediante el modelo Rasch se crean escalas de medición de las posibles respuestas de los estudiantes para cada nivel de dificultad en que se clasifica cada pregunta o ítem.
} 


\section{- Escalas de competencia}

La evaluación PISA no solo reporta el rendimiento de los estudiantes como un puntaje general, sino también en términos del contenido evaluado, ubicando a los estudiantes en una escala que describe lo que estos saben y pueden hacer.

A estas escalas se las llama "escalas de competencia" en lugar de "escalas de desempeño", ya que reportan lo que los estudiantes saben y pueden hacer a ciertos niveles en lugar de lo que estos hicieron en la evaluación (una única ocasión). Se establece de esta manera, ya que con la evaluación PISA se procura obtener el nivel de competencia general del estudiante, y no el rendimiento mostrado en una prueba específica.

Los expertos del programa PISA definieron siete niveles de competencia en lectura, que van desde el nivel $1 \mathrm{~b}$-que implica la realización de tareas elementales que requieren de habilidades muy básicas en lectura- hasta el nivel 6, que involucra tareas de lectura sofisticadas que por lo general solo son capaces de completarlas lectores altamente competentes. En el programa PISA se considera al nivel 2 como el nivel básico de competencias, en el que los estudiantes comienzan a demostrar habilidades en lectura que les permitirán participar eficazmente en la vida productiva.

En Chile y Costa Rica, cerca de la tercera parte de los estudiantes presentan niveles de competencia en lectura inferiores al nivel considerado como básico (nivel 2), y otra tercera parte se ubican en dicho nivel. Por lo tanto, uno de cada tres estudiantes no posee las herramientas cognitivas básicas en el área de lectura para afrontar en el futuro los retos de la sociedad y otro de los tres apenas posee las habilidades básicas.

\section{IV}

\section{Análisis de los resultados de la evaluación PISA para Costa Rica}

\section{Construcción de la muestra utilizada en la prueba PISA}

La muestra de la prueba PISA está diseñada sobre una población de estudiantes cuyas edades fluctúan entre los 15 años y tres meses y los 16 años y dos meses, que asisten a las instituciones educativas oficiales y se encuentran cursando un grado equivalente al séptimo o superior para todos los países participantes. Por estimarse conveniente, se hace referencia a la población como estudiantes de 15 años.

El diseño de la muestra para estudios educativos normalmente no se basa en un muestreo aleatorio simple sobre la población objetivo. En su lugar, se utiliza un muestreo en dos etapas: en la primera etapa, una muestra de centros educativos es seleccionada de una lista completa de los colegios que incluyen a la población de estudiantes de interés. En el caso de la prueba PISA, todos los centros educativos son seleccionados con una probabilidad que es proporcional a su tamaño. Asimismo, se maneja como estándar una tasa de participación mínima de los centros del $85 \%$ a objeto de disminuir el sesgo por no respuesta. En el caso de Costa Rica, la tasa de participación efectiva de los colegios muestreados fue del $99,43 \%$.
En la segunda etapa, se selecciona aleatoriamente una muestra de estudiantes con respecto a los centros educativos elegidos. En los estudios realizados por el programa PISA, se selecciona a un mínimo de 35 estudiantes de 15 años de edad por cada centro educativo participante. En Costa Rica, 4.578 estudiantes fueron efectivamente evaluados, lo que da una tasa de participación del $94,45 \%$ (la tasa mínima requerida que se establece en el programa PISA es del $80 \%)^{3}$.

Sobre esta muestra de estudiantes, en el cuadro A.2 del anexo se resumen algunas estadísticas. Respecto de este y de todos los cuadros presentados en este artículo, la desviación estándar se muestra entre paréntesis ${ }^{4}$.

\footnotetext{
${ }^{3}$ El índice de cobertura de la población de adolescentes de 15 años de edad fue de 0,53 puntos, siendo esta una limitación de los estudios que utilizan estas bases de datos, ya que se excluye a la población de adolescentes de 15 años de edad que no se encuentran cursando la educación oficial, o que desertaron, nunca ingresaron o fueron excluidos del sistema educativo, lo que podría generar un sesgo de selección. Este estudio es representativo únicamente para estudiantes que asisten a la educación oficial diurna de colegios académicos y técnicos rurales y urbanos.

4 Todos los promedios presentados en este artículo corresponden a promedios ponderados en que se utilizó la ponderación final del estudiante (W_FSTUWT) de las bases de datos del programa PISA. Para el cálculo de la desviación estándar se emplea el método recomendado
} 
Del total de estudiantes evaluados, un $53 \%$ son mujeres y el $85 \%$ se encontraban matriculados en colegios públicos. Aunque el nivel oficial que debe cursar un individuo de 15 años de edad en Costa Rica es el noveno año, fueron evaluados estudiantes que se encontraban en casi todos los niveles, pero predominantemente en noveno y décimo años (75\%). La proporción de estudiantes que cursan el séptimo año se debe en su mayoría a alumnos que perdieron uno o más años en la escuela o en el colegio.

\section{Análisis de las brechas educativas}

Las brechas en educación pueden presentarse debido a diferentes condiciones; entre las más comunes se encuentran las brechas de género, por zona o sector al que pertenece el centro educativo y por el tipo de centro educativo.

En el cuadro 1 se muestran las brechas en el rendimiento de los estudiantes en la prueba PISA por género y tipo de centro educativo para cada una de las áreas evaluadas. No se presentan resultados por zona, ya que en el programa PISA no se recolecta información sobre la zona de residencia de los estudiantes.

por el programa de réplica repetida y equilibrada, utilizando otros 80 ponderadores (W_FSTR1, W_FSTR2...,W_FSTR80) siguiendo los capítulos 7 y 8 del Manual de análisis de datos del programa PISA (OCDE, 2009).

\section{- Brechas de género}

En la evaluación del año 2009, las mujeres obtuvieron en promedio mejores resultados que los hombres en el área de lectura en todos los países participantes. Según se muestra en el cuadro 1, la brecha de género en lectura en Costa Rica es de 14 puntos. Sin embargo, Costa Rica se ubica en el grupo de países donde esta diferencia es de las más reducidas.

A diferencia de lo que sucede en lectura, en las áreas de matemáticas y ciencias las brechas son desfavorables para las mujeres: -26 puntos en matemáticas $\mathrm{y}-17$ en ciencias.

\section{- Brechas por tipo de centro educativo}

$\mathrm{Al}$ analizar los resultados de los estudiantes por el tipo de centro educativo al que asisten se observan las mayores disparidades. En las tres áreas, los estudiantes de los colegios privados promediaron mejores resultados que aquellos de colegios públicos. En lectura, la brecha fue de 86 puntos, en matemáticas de 79 puntos y en ciencias de 84 puntos.

De acuerdo con la ubicación de los estudiantes de colegios privados en las escalas de competencia, se observa que solo un $5 \%$ de ellos en la evaluación de lectura, el $16 \%$ en matemáticas y un $8 \%$ en ciencias se encuentran en los niveles de competencia inferiores al nivel básico, mientras que en el sector público los

CUADRO 1

Costa Rica: puntuación promedio y brechas educativas en la prueba PISA 2009, por área de evaluación

\begin{tabular}{|c|c|c|c|c|c|c|}
\hline \multirow{2}{*}{ Categoría } & \multicolumn{2}{|c|}{ Lectura } & \multicolumn{2}{|c|}{ Matemáticas } & \multicolumn{2}{|c|}{ Ciencias } \\
\hline & Puntuación & Brecha & Puntuación & Brecha & Puntuación & Brecha \\
\hline \multirow[t]{2}{*}{ General } & 443 & & 409 & & 430 & \\
\hline & $(3,2)$ & & $(3,0)$ & & $(2,8)$ & \\
\hline
\end{tabular}

Género

\begin{tabular}{lcccccc} 
Mujeres & 449 & 14 & 397 & -26 & 423 & -17 \\
Hombres & $(3,0)$ & & $(3,1)$ & & $(2,8)$ & 440 \\
& 435 & $(2,3)$ & 423 & $(2,1)$ & $(2,4)$ & $(3,3)$ \\
\hline
\end{tabular}

Tipo de centro educativo

\begin{tabular}{lcccccc} 
Público & 429 & -87 & 397 & -79 & 418 & -84 \\
& $(3,1)$ & & $(2,8)$ & & $(2,7)$ & 502 \\
Privado & 516 & $(6,5)$ & 476 & $(6,3)$ & $(6,2)$ \\
& $(5,7)$ & & $(5,8)$ & & $(5,7)$ & \\
\hline
\end{tabular}

Fuente: elaboración propia sobre la base de datos de la evaluación internacional PISA 2009.

Nota: desviación estándar entre paréntesis. 
porcentajes corresponden al 38\% en lectura, $61 \%$ en matemáticas y $45 \%$ en ciencias.

En consecuencia con lo anterior, en los cuatro niveles más altos en las escalas de competencia los colegios privados ubicaron al $73 \%$ de sus estudiantes en lectura ( $25 \%$ en el caso de los públicos), al $50 \%$ de ellos en matemáticas (11\% los colegios públicos) y al $61 \%$ de los alumnos en ciencias (16\% en el sector público).

\section{Las diferencias entre los estudiantes de colegios públicos y privados}

En la encuesta se indaga sobre algunos bienes que se pueden encontrar disponibles en el hogar y que pueden facilitar el proceso de aprendizaje del estudiante; por ejemplo, si el estudiante posee habitación propia o un escritorio para estudiar. En ambos casos, cerca del $90 \%$ de los estudiantes del sector privado disponen de los dos bienes, mientras que entre los del sector público las cifras están por debajo del $70 \%$ (véase el cuadro A.3 del anexo).

Otro de los factores que se menciona en la literatura como determinante del rendimiento educativo es el acceso a las tecnologías de la información y las comunicaciones (TIC). En este caso, la brecha entre estudiantes del sector público y privado es elevada: casi el $100 \%$ de los estudiantes costarricenses que asisten a colegios privados cuentan con computadoras para estudiar y el $91 \%$ con acceso a internet en sus hogares, mientras que poco más de la mitad de sus contrapartes del sector público tienen computadoras $(56 \%)$ y tan solo la tercera parte de ellos $(31 \%)$ cuentan con acceso a internet en su hogar.
Al analizar las características laborales de los padres de los estudiantes de ambos sectores educativos, se observa que el porcentaje de padres de los estudiantes de colegios privados que laboran a tiempo completo es más alto que entre los padres de los estudiantes de colegios públicos ( $83 \%$ comparado con $70 \%$ ); igual sucede con las madres ( $45 \%$ en comparación con $25 \%$ ). Asimismo, el porcentaje de las madres que no trabajan es mayor con respecto a los estudiantes que asisten a colegios públicos (41\% comparado con $62 \%$ ) (véase el cuadro A.4 del anexo).

Con respecto al nivel educativo, mientras que el porcentaje de padres de estudiantes del sector privado, que como máximo concluyeron sus estudios primarios, se sitúa alrededor del $12 \%$, los padres de estudiantes del sector público en esta condición superan el 50\%, de los cuales un $13 \%$ no concluyeron ningún nivel educativo. Asimismo, aproximadamente el 15\% de los padres de estudiantes del sector público poseen estudios universitarios, mientras que esta cifra ronda el $60 \%$ en el caso de los padres que envían a sus hijos a colegios privados.

Dos de las características que más diferencian a los sectores son el porcentaje de profesores que poseen un título de licenciatura o superior (37\% en el sector público y $50 \%$ en el privado) y la disponibilidad de computadoras en los centros educativos, ya que en el sector público, por cada 5 alumnos de 15 años de edad hay una computadora disponible para estudiar, mientras que en el sector privado por cada 5 estudiantes existen 4 computadoras para fines educativos.

\section{V}

\section{La función de producción educativa}

\section{Consideraciones básicas}

La función de producción educativa (FPE) es la herramienta más utilizada por los investigadores para medir la calidad en la educación y sus determinantes. Todd y Wolpin (2003) exponen consideraciones fundamentales sobre la FPE, las que se resumen a continuación.

Las teorías sobre la calidad en la educación y sus determinantes se basan en una analogía entre el proceso de adquisición de conocimientos y habilidades en los seres humanos y el proceso de producción de una empresa: la combinación de varios factores de producción o insumos mediante una tecnología de producción genera un producto o resultado. En el caso de las funciones de producción educativas, la combinación de estos factores tiene como resultado el rendimiento cognitivo, medido a través de una determinada prueba o evaluación.

Para poder realizar la medición de la calidad de la educación con la FPE es necesario contar con el conjunto completo de información sobre los factores a los que cada individuo se ha visto expuesto desde el momento de su nacimiento y que podrían repercutir en su rendimiento cognitivo en un momento dado. En otras palabras, es necesario disponer de datos pasados y presentes respecto 
de los factores familiares y escolares, así como de la dotación inicial de conocimientos del individuo, para poder evaluar su efecto en el rendimiento cognitivo.

Usualmente, las bases de datos para analizar la FPE disponen de información acerca de los factores escolares y familiares, pero medidos en un momento en el tiempo; sin embargo, y sobre todo en el caso de los factores familiares, la información es contemporánea, es decir, no se cuenta con la información sobre los factores en momentos previos a la evaluación. Por tal razón, en varios estudios en que se utiliza la FPE a menudo los factores que afectan a la educación se tratan en etapas tempranas de crecimiento de los individuos como valores inobservables, realizando supuestos sobre la base de los cuales estos valores inobservables pueden ser obviados o eliminados.

\section{Modelo general}

En el modelo general utilizado para analizar el rendimiento cognitivo se asume que el rendimiento de un individuo, medido por una evaluación particular a una edad específica, es el resultado de un proceso acumulativo de adquisición de conocimiento.

Sea $T_{i j a}$ la medida del rendimiento del individuo " $i$ " que reside en el hogar " $j "$ a la edad de " $a$ " años. Se llama $F_{i j a}$ al vector de factores familiares a una edad dada y $S_{i j a}$ al vector de factores escolares. Los vectores que contienen la historia acumulada de cada uno de los factores a la edad de " $a$ " años son $F_{i j}(a)$ y $S_{i j}(a)$. La dotación inicial de capacidades del individuo es denotada como $u_{i j 0}$. Tomando en cuenta la medición del error en el resultado de las pruebas $\left(\epsilon_{i j a}\right)$, la función de producción educativa se expresa de la siguiente manera:

$$
T_{i j a}=T_{a}\left[F_{i j}(a), S_{i j}(a), u_{i j 0}, \epsilon_{i j a}\right]
$$

Sin embargo, la implementación empírica de este modelo presenta dos problemas:

i) la dotación genética de capacidades del individuo no es medible: es inobservable;

ii) los conjuntos de datos en los factores se encuentran incompletos: la historia de los factores es incompleta o se presentan factores perdidos.

Para lidiar con estos problemas, en la literatura en funciones de producción se presentan tres maneras para especificar el modelo, cada una con diferentes variantes y supuestos (Todd y Wolpin, 2003, págs. F16, F27).

La especificación que fue utilizada en esta investigación, la especificación contemporánea, se basa en el supuesto de que la medida final del rendimiento cognitivo a través de una prueba o evaluación se relaciona únicamente con los datos contemporáneos de los factores familiares y escolares.

Teniendo presente este supuesto en el momento de analizar los resultados, la función de producción educativa se expresa en la siguiente ecuación:

$$
\left.T_{i j a}=T_{a}\left(F_{i j a}, S_{i j a}\right)+\epsilon_{i j a}^{\prime}\right]
$$

Donde $\epsilon_{i j a}^{\prime}$ es un término de error aditivo. En esta especificación, en el término de error se incluyen todos los factores omitidos (la historia de los factores pasados, la dotación inicial de capacidades y la medida del error).

Si bien es cierto que esta especificación posee algunas limitaciones, ello no niega el poder explicativo de la función, ya que hasta la fecha no se dispone de muchas investigaciones en el tema de la calidad de la educación en Costa Rica y, por lo tanto, se espera que esta investigación pueda aportar resultados prácticos para orientar futuras investigaciones.

\section{Determinantes de la educación}

En las investigaciones que utilizan funciones de producción educativas normalmente se clasifican todos los factores que podrían afectar al proceso de aprendizaje de un estudiante dentro de tres categorías generales: factores familiares y del estudiante, factores escolares y factores institucionales y de política educativa. A continuación, se procede a enumerar algunos de ellos de acuerdo con lo recopilado por Vegas y Petrow (2007).

\section{— Factores familiares y del estudiante}

Estos son los factores que el estudiante trae consigo antes de ingresar al sistema educativo, y se refieren tanto a características propias del estudiante como del entorno familiar. La edad a la que el estudiante ingresa a la educación primaria y la preparación recibida antes de ingresar a la primaria son dos de los factores que han cobrado relevancia (Urzúa y Veramendi, 2011).

En recientes investigaciones se ha evaluado el efecto que tiene en el rendimiento de un estudiante la interacción con sus pares (peer effect ${ }^{5}$ ). No obstante, son los factores familiares y el apoyo que el niño recibe en su hogar los que usualmente tienen los mayores efectos en la explicación del rendimiento cognitivo. El estatus socioeconómico de la familia del estudiante y

\footnotetext{
5 Epple y Romano (1998); Mizala y Romaguera (2002).
} 
el ingreso familiar han sido las variables más utilizadas como variables sustitutivas (proxy) de estos factores.

Sin embargo, estas variables por sí solas no pueden dar cuenta de la dinámica no observable del hogar y de lo que realmente está pasando en su interior, como es el caso de la participación y el apoyo de los padres en la educación de sus hijos. Por esto, resulta importante no subestimar la influencia de las acciones de los padres en el hogar en el rendimiento de sus hijos (Urzúa y Veramendi, 2011, pág. 83).

\section{- Factores escolares}

Estos son las dotaciones y los recursos que ofrecen los centros educativos y que terminan afectando al nivel de rendimiento de un estudiante. Pueden clasificarse en dos categorías: las características del centro educativo y las características de los profesores.

En el caso de las características de los centros educativos, las variables más utilizadas para captar su efecto son la cantidad de libros que ofrecen los centros educativos, las bibliotecas, y otras facilidades de la infraestructura propia del centro educativo, como salas de estudio para los alumnos, tamaño y comodidades del aula, equipamiento tecnológico, entre otros. Sin embargo, en algunos estudios se ha demostrado que la incidencia de las TIC en el rendimiento educativo es limitada o prácticamente nula (Cristia, Czerwonko y Garofalo, 2010; Cristia y otros, 2012).

Por otra parte, los profesores pueden influir significativamente en el rendimiento de los estudiantes, ya que son ellos quienes participan de manera directa y continua en el proceso de aprendizaje de sus alumnos, y por ende, un profesor poco calificado o que recurre a métodos de enseñanza inefectivos puede afectar negativamente al rendimiento de los estudiantes, o viceversa.

\section{— Factores institucionales y de política educativa}

La estructura organizacional de un sistema educativo juega un papel relevante en el aprendizaje de los estudiantes. La manera en que estén distribuidas las responsabilidades de los tomadores de decisiones en temas como financiamiento, gasto, movilidad del personal (contratar y despedir profesores) e independencia para establecer las metodologías de enseñanza, entre otros, son factores institucionales que pueden afectar al rendimiento de los alumnos.

Otro factor institucional que ha cobrado relevancia es la selectividad académica (tracking). Esta se refiere a la movilización y ubicación de los estudiantes en diferentes centros de enseñanza de acuerdo con su nivel educativo ${ }^{6}$. No obstante, esta no es una política educacional que se aplique en Costa Rica.

\section{Literatura sobre la función de producción educativa (FPE)}

El Informe Coleman (Coleman, 1966) fue uno de los primeros estudios desarrollado sobre este tema y aún tiene una influencia significativa en las investigaciones acerca del rendimiento educativo. En él se sugiere que las diferencias en los factores escolares tienen poco que ver con las diferencias en rendimiento, mientras que los factores familiares son más importantes.

Otras investigaciones han llegado a conclusiones similares: Hanushek (1986) realiza una revisión de los estudios completados hasta mediados de la década de 1980, encontrando que la evidencia del efecto del nivel de gasto por estudiante $u$ otros factores escolares en el rendimiento educativo es extremadamente débil y desaparece cuando se toman en cuenta las diferencias en los factores familiares. Más recientemente, Lee y Barro (2001), al utilizar la evaluación del Tercer Estudio Internacional de Matemáticas y Ciencias (TIMss, por sus siglas en inglés) para un gran número de países, muestran que las características familiares (ingreso y educación de los padres) tienen un gran efecto en el rendimiento educativo. Formichella (2011), en un estudio realizado para la Comisión Económica para América Latina y el Caribe (CEPAL) con datos de los resultados respecto de estudiantes argentinos en la prueba PISA de 2006, afirma que los individuos que habitan en un hogar con mejor clima educativo y mayores recursos relacionados con la educación obtienen resultados académicos más elevados.

$\mathrm{Al}$ contrario de lo que sucede con los factores familiares, la evidencia acerca de la influencia de los factores escolares es mixta y en muchos casos no concluyente (Greenwald, Hedges y Laine, 1996; Kremer, 1995; Card y Krueger, 1996).

\section{Técnica de descomposición de Fields}

En la literatura sobre desigualdad, especialmente de ingresos, se han desarrollado diferentes métodos de descomposición (Shorrocks 1980, 1982 y 1984; Fields, 2003; Morduch y Sicular 2002). La desigualdad puede

\footnotetext{
${ }^{6}$ Hanushek y Woessmann (2005 y 2010).
} 
ser descompuesta por subgrupos, fuentes de ingreso, factores causales y por características sociodemográficas; además, puede descomponerse en diferentes niveles de agregación. Heshmati (2004) presenta un resumen de los diferentes métodos de descomposición. En este artículo se optó por utilizar la técnica de descomposición de Fields.

Dicha técnica se utiliza para descomponer el aporte de cada variable explicatoria de la desigualdad total de la variable dependiente. Fue desarrollada por Gary S. Fields (2003), y usualmente se basa en la ecuación de salarios de Mincer para descomponer el aporte de diversas variables a la explicación de la desigualdad de los ingresos. En el caso del rendimiento de los estudiantes medido en la prueba PISA, la ecuación (2) se construye de la siguiente manera para presentar la descomposición de Fields:

$$
\ln \left(T_{i a}\right)=\sum_{j=1}^{n} C_{i a} * X_{i a j}+\varepsilon_{i a}=\sum_{j=1}^{n} C_{i a} * Z_{i a j}
$$

donde: $\ln \left(T_{i a}\right)$ es el logaritmo natural del valor plausible. $X_{i a j}$ son las variables $j$ vinculadas a la persona $i$ a la edad de $a$ años.

$C_{i a}$ son los coeficientes que acompañan a cada variable, y $\varepsilon_{i a}$ es la parte de la variación del rendimiento entre los estudiantes que no puede ser explicada por la variación entre las variables incluidas en la ecuación.

Aplicando la varianza a cada lado de la ecuación anterior y después de algunos cálculos matemáticos, se obtiene lo siguiente:

$$
1=\frac{\sum_{j} \operatorname{Cov}\left[C_{j} Z_{j}, \ln (T)\right]}{\operatorname{Var} \ln (T)} \cong \sum_{j} S_{j}
$$

donde cada $S_{j}$ es llamado "peso relativo del factor en la variabilidad" y está dado por:

$$
S_{j}=\frac{\operatorname{Cov}\left(C_{j} Z_{j}, \ln (T)\right)}{\operatorname{Var}(\ln (T))}
$$

La ecuación anterior (5) se puede interpretar como la medida en la proporción de la varianza del logaritmo del valor plausible que es explicada por cada variable regresora ${ }_{j}$. Cabe hacer notar que si el $S_{j}$ del residuo es excluido, la sumatoria de los pesos relativos es exactamente igual a la medida de bondad de ajuste de la regresión $\left(\mathrm{R}^{2}\right)$.

A través de esta ecuación se logra estimar el peso relativo de cada variable incluida dentro del modelo en la explicación del rendimiento cognitivo de los estudiantes.

\section{Limitaciones del modelo}

La técnica de descomposición de Fields, al igual que otras técnicas de descomposición paramétricas, mantiene como desventaja la imposición de una forma funcional al proceso de acumulación de conocimientos, mientras que los métodos no paramétricos o semiparamétricos evitan imponer una forma funcional determinada (aunque su cálculo puede ser en extremo complejo) (Contreras y Gallegos, 2011, pág. 29).

Una limitación de la forma funcional utilizada es que no incorpora la consideración de la dependencia de las observaciones dentro de cada grupo, al estar en presencia de una estructura multinivel. Un modelo lineal podría no ser la mejor herramienta para medir la relación entre el rendimiento en la prueba PISA y las variables seleccionadas.

Sin embargo, la principal razón por la que se optó por el uso de esta técnica de descomposición radica en que con ella se puede cuantificar el efecto de cada una de las regresoras en la desigualdad educativa, al permitir la inclusión de variables dicotómicas, con lo que se puede descomponer el efecto aislado de cada variable explicativa. 


\section{VI}

\section{Resultados a nivel nacional}

Se utilizó como variable dependiente el resultado obtenido por los estudiantes en la prueba PISA ${ }^{7}$. La función de producción educativa fue estimada tanto para el modelo completo, que incluye a todos los estudiantes dentro de la muestra, como para dos submuestras: estudiantes de colegios públicos y estudiantes de colegios privados. Debido a limitaciones de espacio, solo se muestran los coeficientes del modelo general en el cuadro A.5 del anexo ${ }^{8}$.

Lo primero que debe ser aclarado es que el $41 \%$ de las diferencias en el rendimiento cognitivo de los estudiantes se explica por las variables incluidas en el modelo para la evaluación de lectura, lo que quiere decir que el 59\% corresponde al término de error de la ecuación y se refiere a variables que no fueron incluidas en el modelo. Para las evaluaciones de matemáticas y ciencias, el ajuste del modelo fue del $43 \%$ y $37 \%$, respectivamente.

Por otra parte, algunas variables resultaron no ser significativas en la explicación de las disparidades de rendimiento, como el estatus ocupacional más alto de los padres, el disponer de internet en el hogar o la cantidad de computadoras disponibles para estudiar en los centros educativos.

Al aplicar la técnica de descomposición de Fields para el modelo que incluye a todos los estudiantes, se obtienen los siguientes resultados (véase el cuadro A.6 del anexo).

Para las tres áreas, los factores familiares y del estudiante son los que explican en mayor medida las diferencias en el rendimiento educativo de los estudiantes evaluados en la prueba PISA.

Si bien es cierto que las mujeres obtuvieron mejores resultados que los hombres en lectura, esto no se debe

\footnotetext{
${ }^{7}$ La variable dependiente corresponde al logaritmo natural del valor plausible. Por cada área de evaluación fueron estimadas 405 regresiones mediante mínimos cuadrados ponderados, utilizando los ponderadores de la base de datos del programa PISA. La regresión también fue estimada empleando el valor plausible como variable dependiente sin aplicar el logaritmo natural, por lo que se pudo corroborar que el ajuste del modelo y la significancia de las variables no variaron cuando se aplicó el logaritmo natural al valor plausible.

8 De los 4.578 estudiantes evaluados, la muestra se redujo a 4.351 observaciones debido a la existencia de datos perdidos. Se perdieron 227 observaciones (5\% del total), concentradas especialmente en las variables familiares y personales. Algunas variables no fueron incluidas en el modelo final para evitar una mayor pérdida de datos.
}

a la condición de género, ya que el peso de la variable Mujer en la función de producción educativa de lectura no alcanza al 1\%. En matemáticas y ciencias sí pareciera existir mayor evidencia de que la condición de género del estudiante incide en los resultados de las evaluaciones, especialmente en matemáticas $(4,3 \%)$.

El modelo presenta evidencia de que es el año que esté cursando el estudiante en el colegio lo que más afecta a las diferencias en el rendimiento educativo. Con el fin de analizar este hecho, en el cuadro 2 se presenta la puntuación promedio por año de colegio cursado:

CUADRO 2

Costa Rica: puntuación promedio en PISA 2009 de los estudiantes de 15 años, según año cursado

\begin{tabular}{lrrrrrr}
\hline Año & \multicolumn{2}{c}{ Lectura } & \multicolumn{2}{c}{ Matemáticas } & \multicolumn{2}{c}{ Ciencias } \\
\hline Séptimo & 344 & $(5,3)$ & 330 & $(4,5)$ & 346 & $(4,6)$ \\
Octavo & 392 & $(4,1)$ & 367 & $(3,5)$ & 387 & $(3,5)$ \\
Noveno & 442 & $(2,6)$ & 409 & $(2,9)$ & 433 & $(2,7)$ \\
Décimo & 483 & $(3,7)$ & 443 & $(3,6)$ & 462 & $(3,1)$ \\
Undécimo & 498 & $(15,9)$ & 453 & $(19,0)$ & 487 & $(19,7)$ \\
\hline
\end{tabular}

Fuente: elaboración propia sobre la base de datos de la evaluación internacional PISA 2009.

Nota: desviación estándar entre paréntesis.

Respecto de cada año superior la puntuación se incrementa consistentemente. En el modelo no fue incluida la variable de la edad de los estudiantes, debido a que el rango de edad en el programa PISA es muy corto y podría no ser suficiente para captar el efecto deseado. Sin embargo, se incluyeron cinco variables que clasifican a cada estudiante en el año de colegio que cursaban en el momento en que se realizó la evaluación, lo que capta de mejor manera a los alumnos de acuerdo con su rendimiento educativo promedio esperado: es más probable que aquellos que cursan noveno y décimo de 15 años de edad no hayan repetido años escolares y que además posean conocimientos en lectura, ciencias y matemáticas que les permitan aumentar sus habilidades y, por ende, su rendimiento educativo. Por el contrario, la mayoría de los estudiantes que cursaban séptimo y octavo en el momento de la evaluación habían repetido algún año escolar y poseían además un nivel de conocimientos 
menor que el de aquellos ubicados en niveles educativos superiores, lo que los pone en desventaja con respecto a estos en el rendimiento de la prueba.

La prueba PISA está diseñada para evaluar capacidades y habilidades y no tanto conocimientos específicos; sin embargo, los resultados sugieren que los estudiantes de niveles más avanzados obtienen mayores puntajes y que esta característica es la que más incide en la explicación de las diferencias en rendimiento educativo después de controlar por los demás factores (género, nacionalidad, bienes en el hogar, sector educativo, entre otros). El año de colegio al que asiste el estudiante explica un 20,9\% de la desigualdad de los rendimientos en la evaluación PISA enfocada en lectura.

Los resultados indican que repetir un año académico no soluciona las deficiencias en las habilidades y capacidades de los estudiantes, lo que es necesario para obtener un buen resultado en la evaluación. Por otra parte, los conocimientos adquiridos en cada año adicional podrían incidir en el análisis y las respuestas dadas por los estudiantes en la evaluación, otorgando mayor ventaja a aquellos que cursan niveles superiores.

Al medir los factores escolares, dos variables se destacan por sobre las demás: el tipo de colegio (público o privado) y los recursos educativos presentes en los colegios. Sobre el primero se centra el interés de este artículo: medir cómo repercute en el rendimiento escolar el asistir a un colegio público o a uno privado, según las diferencias en calidad educativa ofrecida en ambos tipos de colegio. En lectura, un 4,11\% de la variabilidad del rendimiento se explica por esta variable (véase el cuadro A.6).

Por lo tanto, el aporte del tipo de colegio - como determinante de la desigualdad de los rendimientos en la evaluación PISA- no es tan contundente como para afirmar que la brecha en el rendimiento escolar de los estudiantes que asisten a colegios públicos y privados se debe principalmente a una mayor calidad educativa en el sector privado. En el caso de matemáticas, esta variable explica un 3,3\% de la variabilidad en los resultados de la evaluación PISA; y en ciencias, el 6\%.

La calidad de los recursos educativos en los colegios figura como la segunda variable en importancia en el grupo de factores escolares: las diferencias en la calidad de recursos explica cerca de un $4 \%$ en las tres áreas. Los factores institucionales apenas afectan a los resultados de los estudiantes.

En síntesis, del modelo general se destaca que son los factores familiares y las características del estudiante los que explican en mayor proporción las diferencias en el rendimiento de los jóvenes evaluados en la prueba PISA, lo que era de esperar debido a la evidencia presentada por otras investigaciones de este tipo.

Por último, la técnica de descomposición de Fields fue también aplicada a la muestra de estudiantes por sector educativo (véase el cuadro A.7 del anexo). En el caso de los alumnos que asisten a colegios públicos, las variables incluidas en el modelo explican en menor medida las diferencias analizadas: $29 \%$ en lectura, $34 \%$ en matemáticas y $27 \%$ en ciencias. Los factores familiares y del estudiante continúan explicando la mayor parte de la variabilidad de los rendimientos $(27 \%, 29 \%$ y $24 \%$ en lectura, matemáticas y ciencias, respectivamente), sobre todo el año de colegio que cursaban los estudiantes. Con respecto a los factores escolares, el peso de estos para explicar las diferencias en rendimientos disminuye.

En cuanto a las diferencias en el rendimiento de los estudiantes que asisten a colegios privados, las variables del modelo ajustan de una mejor manera (54\%, $52 \%$ y $51 \%$ en lectura, matemáticas y ciencias, respectivamente), sobre todo porque en tal caso los factores institucionales sí resultan ser relevantes para explicar estas diferencias.

En lectura, el género de los estudiantes no afecta a los resultados (al igual que en el modelo general), pero en matemáticas y ciencias esta variable resulta mucho más relevante.

Las diferencias en los bienes presentes en los hogares de los estudiantes que asisten a colegios privados también aportan en la explicación $(7 \%, 5 \%$ y $5 \%$ en lectura, matemáticas y ciencias, respectivamente), mientras que en matemáticas y lectura, las diferencias por año de colegio cursado son menos relevantes $(9 \%$ y $11 \%$, respectivamente) que en el área de lectura (16\%).

En los factores escolares, los colegios privados con más y mejores libros, computadoras y laboratorios explican el 7\%, $9 \%$ y $5 \%$ de las diferencias en los resultados de los estudiantes de este sector en lectura, matemáticas y ciencias, respectivamente. Esta situación se presenta debido a que no todos los colegios privados de Costa Rica poseen la misma dotación de recursos, y la variabilidad en este aspecto es mucho mayor que en el caso de los colegios públicos, donde existe mayor similitud en la dotación de recursos disponibles.

Para finalizar, los factores institucionales adquieren importancia en el caso de los colegios privados debido a dos razones: primero, en estos colegios existe mayor libertad para implementar políticas que en los colegios públicos, donde las políticas institucionales son estandarizadas para todo el sector y, segundo, la participación de los padres en la administración escolar tiene un efecto positivo en el aprendizaje. Esta última 
idea se basa en el supuesto de que en las escuelas y colegios privados los padres tienden a participar más en el proceso educativo, ya que podrían estar interesados en examinar el servicio por el que están pagando.

En las tres áreas de evaluación, el factor institucional que más afecta al resultado es el de las actividades extracurriculares que el colegio ofrece a sus estudiantes,

\section{VII}

\section{Conclusiones}

El Programa Internacional de Evaluación de Estudiantes (PISA) se ha enfocado en la medición de la calidad de la educación en diversos países alrededor del mundo, lo que permite a las naciones y a sus ciudadanos conocer el nivel relativo en el que se encuentra la calidad de sus sistemas educativos. El hecho de que Costa Rica participe en este proyecto es un avance importante, ya que permite a los encargados de elaborar las políticas educativas del país formarse una idea bastante clara sobre el estado de la calidad de la educación y de sus principales determinantes.

El programa PISA proporciona una valiosa información para futuras investigaciones sobre la calidad de la educación costarricense. Por ello, es de vital importancia que el Ministerio de Educación Pública continúe participando en el proyecto para poder medir los avances dentro del sistema educativo a través del tiempo y de esta manera evaluar qué tan eficientemente se están utilizando los recursos destinados a la educación de los jóvenes costarricenses.

Los datos indican que en Costa Rica la brecha de rendimiento entre estudiantes de colegios públicos y privados es amplia. Esta brecha obedece sobre todo a las diferencias que existen en los factores familiares, personales o ambos que afectan al estudiante, principalmente el nivel o grado en que este se encuentra en el momento de realizar la prueba. De acuerdo con los datos obtenidos de los cuestionarios, un 26\% de los estudiantes evaluados de los colegios públicos han repetido uno o más años en secundaria, mientras que entre los estudiantes de los colegios privados solo un $10 \%$ han repetido algún año educativo.

Los resultados evidencian claramente la relación entre el grado que cursan los jóvenes y su rendimiento académico. De esta manera se pone de manifiesto el enorme costo que tiene en el rendimiento de los estudiantes como bandas y coros, equipos deportivos, clubes de debate, entre otros $(9,5 \%, 8,8 \%$ y $4,5 \%$ en lectura, matemáticas y ciencias, respectivamente), y en menor medida, los métodos de evaluación de estudiantes y profesores y la independencia en la decisión de distribuir los recursos con que cuenta el colegio y la selectividad académica. repetir uno o más grados. En cierta manera, al repetir un grado se le niega al estudiante el conocimiento de temas esenciales y la oportunidad de desarrollar las destrezas necesarias para mejorar sus habilidades académicas, además de ser un factor de desmotivación. Además, repetir un año podría no ser la mejor estrategia para solventar las deficiencias académicas que presenten los estudiantes, aparte de constituir un factor de desmotivación.

Los recursos de apoyo para la población estudiantil rezagada que permitan disminuir los niveles de repitencia podrían ser esenciales para mejorar la calidad del sistema educativo costarricense. En este sentido, la implementación — desde el año 2009-de las reformas a las normas reguladoras de la promoción y repitencia dentro del sistema educativo público costarricense, podría incidir en el futuro en mejorar el rendimiento de los estudiantes. Antes del año 2009, un estudiante de educación secundaria que reprobara en más de tres materias era obligado a repetir el año lectivo completo, lo que convertía al sistema educativo costarricense en un modelo sumamente excluyente. Sin embargo, con la aplicación de dichas reformas el estudiante no debe repetir el año lectivo completo, sino únicamente las materias reprobadas al mismo tiempo que puede cursar las materias del año lectivo siguiente que no tienen como requisito las reprobadas.

De acuerdo con los resultados de esta investigación, esta reforma educativa podría ser clave para elevar el rendimiento de los jóvenes, y su efecto podría evaluarse en futuras pruebas PISA.

Por otra parte, la diferencia entre la educación impartida en los colegios privados (con más y mejores recursos) y la educación ofrecida en los colegios públicos no tiene un peso tan relevante a la hora de explicar la brecha de rendimiento entre estudiantes de colegios públicos y privados. En otras palabras, los resultados 
demuestran que cuando se trata de explicar la desigualdad en el rendimiento educativo, las diferencias en las condiciones socioeconómicas pesan mucho más que las diferencias en los recursos escolares.

Sería recomendable que además de los cuestionarios aplicados a los estudiantes y directores de colegio por parte de los responsables del programa PISA, se solicite la aplicación de los cuestionarios a los padres de familia, a fin de realizar un análisis más depurado de la influencia de los factores familiares en el rendimiento de los estudiantes, que tienen una enorme importancia a la hora de explicar el rendimiento educativo. Mediante este cuestionario se obtendría valiosa información sobre las características de los hogares de los estudiantes, de actividades de los padres con sus hijos, de discusiones acerca del progreso del joven en su colegio, e incluso del nivel de ingreso del hogar o de la distancia entre el hogar del estudiante y el centro educativo.
Por último, se resalta la calidad de la información y de los datos generados por la prueba PISA, que son lo suficientemente confiables como para que se utilicen en la definición de políticas que disminuyan las brechas educativas entre los jóvenes costarricenses, mejoren la asignación de los recursos públicos e incrementen la calidad de la educación pública. Como fue indicado por el Ministerio de Educación Pública de Costa Rica en su Memoria Institucional 2006-2010 (MEP, 2010), el propósito de participar en pruebas internacionales se orienta al aprovechamiento de la información derivada de las pruebas como insumo para la transformación del proceso de enseñanza y aprendizaje, ya sea por medio de reformas curriculares, estrategias de desarrollo profesional y capacitación continua, o bien mediante la dotación de recursos de apoyo para la mediación y la política educativa en el aula. Para mejorar la educación pública es esencial hacer uso de la información generada en la evaluación PISA. 


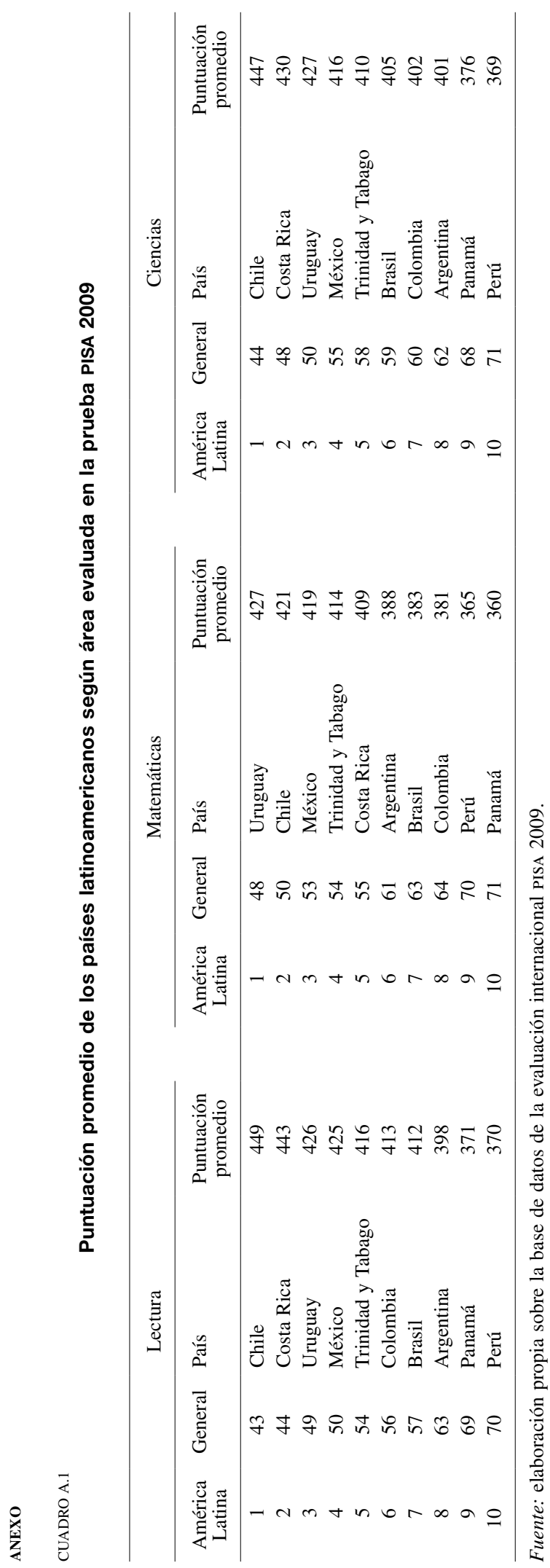


CUADRO A.2

Costa Rica: estadísticas descriptivas de

la evaluación PISA 2009, según categoría

\begin{tabular}{lcc}
\hline Categoría & Porcentaje & $\begin{array}{c}\text { Desviación } \\
\text { estándar }\end{array}$ \\
\hline Género & & \\
$\quad$ Mujeres & 53,0 & $(0,6)$ \\
$\quad$ Hombres & 47,0 & $(0,6)$ \\
Sector educativo al que asiste & & \\
$\quad$ Público & 84,6 & $(1,4)$ \\
$\quad$ Privado & 15,4 & $(1,4)$ \\
Año de colegio que cursa & & \\
$\quad$ Séptimo & 8,5 & $(0,8)$ \\
$\quad$ Octavo & 16,0 & $(1,0)$ \\
$\quad$ Noveno & 34,1 & $(1,2)$ \\
$\quad$ Décimo & 40,9 & $(1,8)$ \\
$\quad$ Undécimo & 0,4 & $(0,1)$ \\
$\quad$ Duodécimo & 0,0 & $(0,0)$ \\
\hline
\end{tabular}

Fuente: elaboración propia sobre la base de datos de la evaluación internacional PISA 2009.

CUADRO A.3

Costa Rica: estadísticas descriptivas de la evaluación por sector educativo, según característica del estudiante o del hogar, 2009

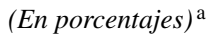

\begin{tabular}{|c|c|c|c|c|}
\hline \multirow{2}{*}{$\frac{\text { Característica }}{\text { Del estudiante }}$} & \multicolumn{2}{|c|}{ Público } & \multicolumn{2}{|c|}{ Privado } \\
\hline & & & & \\
\hline Mujeres & 53,2 & $(0,7)$ & 51,8 & $(1,8)$ \\
\hline Asistió a preescolar & 74,7 & $(1,3)$ & 91,0 & $(2,0)$ \\
\hline Repitió grado en escuela & 18,0 & $(1,2)$ & 4,0 & $(1,4)$ \\
\hline Repitió grado en colegio (III ciclo) & 26,0 & $(1,3)$ & 10,0 & $(1,5)$ \\
\hline \multicolumn{5}{|l|}{ Grupo familiar } \\
\hline Vive con ambos padres & 68,6 & $(1,1)$ & 78,5 & $(1,8)$ \\
\hline Vive solo con madre & 21,2 & $(0,8)$ & 17,1 & $(1,3)$ \\
\hline Vive solo con padre & 2,1 & $(0,3)$ & 2,0 & $(0,7)$ \\
\hline No vive con padres & 8,1 & $(0,5)$ & 2,4 & $(1,3)$ \\
\hline \multicolumn{5}{|l|}{ Bienes del hogar } \\
\hline Habitación propia & 69,5 & $(1,0)$ & 88,2 & $(1,3)$ \\
\hline Escritorio & 60,1 & $(1,3)$ & 93,1 & $(1,5)$ \\
\hline Computadora & 56,5 & $(1,4)$ & 97,3 & $(0,6)$ \\
\hline Internet & 31,0 & $(1,3)$ & 90,9 & $(1,4)$ \\
\hline Arte & 39,0 & $(1,1)$ & 81,1 & $(1,7)$ \\
\hline Automóvil & 45,5 & $(1,1)$ & 86,7 & $(1,9)$ \\
\hline Baño o ducha & 62,8 & $(1,6)$ & 98,0 & $(0,6)$ \\
\hline
\end{tabular}

Fuente: elaboración propia sobre la base de datos de la evaluación internacional PISA 2009.

a El porcentaje corresponde a la proporción de estudiantes que poseen la característica, cumplen con la condición o poseen el bien en su hogar. Nota: desviación estándar entre paréntesis. 


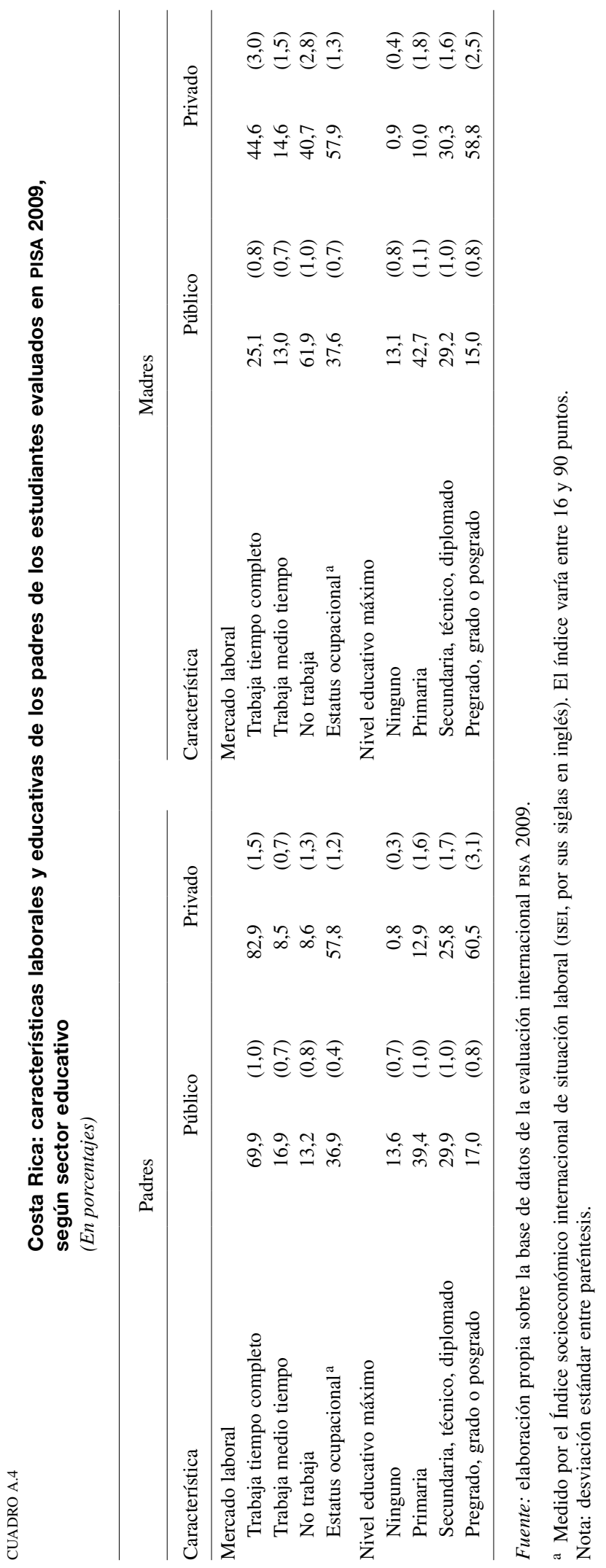


CUADRO A.5

Costa Rica: determinantes del rendimiento educativo.

Coeficientes de la función de producción educativa, 2009

Variable dependiente: logaritmo natural del valor plausible de la evaluación internacional PISA 2009 a

Constante

Factores familiares y del estudiante

Mujer

Nacionalidad costarricense

Asistió a preescolar

Octavo

Noveno

Décimo

Undécimo

Estatus ocupacional

Educación de la madre:

Primaria completa

Secundaria completa

Universitaria

Libros en el hogar:

10 a 25

25 a 100

100 a 200

200 a 500

Más de 500

Índice de posesiones del hogar

Índice de recursos educativos en el hogar

Computadora en el hogar

Internet en el hogar

Efecto de pares (peer effect)

Factores escolares

Características del centro educativo

Colegio público

Colegio único en la zona

Directora

Índice de recursos educativos en el colegio

Computadoras disponibles para estudiar

Aprendizaje condicionado por falta o inadecuado recurso:

$$
\text { Libros }
$$

Laboratorio de ciencias

Computadoras

Características de los profesores

Aprendizaje condicionado por profesores no calificados de:

Selectividad académica (tracking)

Índice de liderazgo escolar

Índice de actividades extracurriculares

Índice de responsabilidad del colegio en:

Currículo y evaluación

Distribución de los recursos
Lectura
Matemáticas
Ciencias

Factores institucionales

Modelo completo

\begin{tabular}{cccccc}
\hline \multicolumn{2}{c}{ Lectura } & \multicolumn{2}{c}{ Matemáticas } & \multicolumn{2}{c}{ Ciencias } \\
\hline 5,732 & $(0,03)$ & 5,805 & $(0,03)$ & 5,802 & $(0,03)$ \\
& & & & & \\
0,019 & $(0,00)$ & $-0,074$ & $(0,00)$ & $-0,054$ & $(0,00)$ \\
$0,007^{\mathrm{t}}$ & $(0,01)$ & $-0,001^{\mathrm{t}}$ & $(0,01)$ & $-0,001^{\mathrm{t}}$ & $(0,01)$ \\
0,017 & $(0,01)$ & 0,021 & $(0,01)$ & 0,011 & $(0,01)$ \\
0,097 & $(0,01)$ & 0,077 & $(0,01)$ & 0,088 & $(0,01)$ \\
0,173 & $(0,01)$ & 0,151 & $(0,01)$ & 0,165 & $(0,01)$ \\
0,248 & $(0,01)$ & 0,223 & $(0,01)$ & 0,222 & $(0,01)$ \\
0,299 & $(0,03)$ & 0,269 & $(0,03)$ & 0,294 & $(0,03)$ \\
$0,000^{\mathrm{t}}$ & $(0,00)$ & $0,000^{\mathrm{t}}$ & $(0,00)$ & $-0,000^{\mathrm{t}}$ & $(0,00)$ \\
& & & & & \\
$0,000^{\mathrm{t}}$ & $(0,01)$ & 0,016 & $(0,01)$ & $0,015^{\mathrm{t}}$ & $(0,01)$ \\
$0,014^{\mathrm{t}}$ & $(0,01)$ & 0,037 & $(0,01)$ & $0,014^{\mathrm{t}}$ & $(0,01)$ \\
$0,018^{\mathrm{t}}$ & $(0,01)$ & 0,041 & $(0,01)$ & 0,025 & $(0,01)$ \\
& & & & & \\
0,015 & $(0,01)$ & $-0,003^{\mathrm{t}}$ & $(0,01)$ & 0,016 & $(0,01)$ \\
0,023 & $(0,01)$ & 0,020 & $(0,01)$ & 0,021 & $(0,01)$ \\
0,037 & $(0,01)$ & $0,021^{\mathrm{t}}$ & $(0,01)$ & 0,043 & $(0,01)$ \\
0,055 & $(0,01)$ & 0,033 & $(0,01)$ & 0,053 & $(0,01)$ \\
0,067 & $(0,03)$ & $0,033^{\mathrm{t}}$ & $(0,03)$ & 0,071 & $(0,03)$ \\
0,009 & $(0,00)$ & 0,010 & $(0,00)$ & $0,002^{\mathrm{t}}$ & $(0,00)$ \\
$-0,011$ & $(0,00)$ & $-0,010$ & $(0,00)$ & $-0,006^{\mathrm{t}}$ & $(0,00)$ \\
0,019 & $(0,01)$ & 0,017 & $(0,01)$ & 0,021 & $(0,01)$ \\
$0,005^{\mathrm{t}}$ & $(0,01)$ & $0,009^{\mathrm{t}}$ & $(0,01)$ & 0,017 & $(0,01)$ \\
0,003 & $(0,01)$ & 0,004 & $(0,01)$ & 0,003 & $(0,01)$ \\
& & & & & \\
& & & & &
\end{tabular}

\begin{tabular}{|c|c|c|c|c|c|}
\hline$-0,051$ & $(0,01)$ & $-0,039$ & $(0,01)$ & $-0,074^{t}$ & $(0,01)$ \\
\hline $0,004^{\mathrm{t}}$ & $(0,01)$ & $-0,006^{t}$ & $(0,01)$ & $-0,002$ & $(0,01)$ \\
\hline 0,011 & $(0,01)$ & 0,015 & $(0,00)$ & $-0,001^{t}$ & $(0,01)$ \\
\hline 0,017 & $(0,00)$ & 0,018 & $(0,00)$ & 0,018 & $(0,00)$ \\
\hline $0,000^{t}$ & $(0,00)$ & $0,000^{t}$ & $(0,00)$ & $-0,000^{t}$ & $(0,00)$ \\
\hline $0,002^{\mathrm{t}}$ & $(0,01)$ & $-0,008^{t}$ & $(0,01)$ & $-0,013^{t}$ & $(0,01)$ \\
\hline$-0,008^{t}$ & $(0,01)$ & $-0,005^{t}$ & $(0,01)$ & $0,014^{\mathrm{t}}$ & $(0,01)$ \\
\hline $0,004^{\mathrm{t}}$ & $(0,01)$ & $-0,008^{t}$ & $(0,01)$ & $0,015^{\mathrm{t}}$ & $(0,01)$ \\
\hline $0,010^{t}$ & $(0,01)$ & $0,010^{t}$ & $(0,01)$ & $0,003^{t}$ & $(0,01)$ \\
\hline$-0,011^{\mathrm{t}}$ & $(0,01)$ & $-0,019$ & $(0,01)$ & $-0,017^{t}$ & $(0,01)$ \\
\hline $0,003^{t}$ & $(0,01)$ & 0,026 & $(0,01)$ & $0,015^{t}$ & $(0,01)$ \\
\hline 0,001 & $(0,01)$ & 0,002 & $(0,01)$ & $0,002^{\mathrm{t}}$ & $(0,01)$ \\
\hline$-0,009$ & $(0,00)$ & $-0,009$ & $(0,00)$ & $-0,001^{t}$ & $(0,00)$ \\
\hline 0,019 & $(0,00)$ & 0,013 & $(0,00)$ & 0,016 & $(0,00)$ \\
\hline $0,001^{t}$ & $(0,00)$ & $-0,002^{t}$ & $(0,00)$ & $-0,001^{t}$ & $(0,00)$ \\
\hline$-0,011$ & $(0,01)$ & $-0,014$ & $(0,01)$ & $-0,005^{t}$ & $(0,01)$ \\
\hline \multicolumn{2}{|c|}{4351} & & \\
\hline & \multicolumn{2}{|c|}{0,429} & & \\
\hline
\end{tabular}

$\mathrm{N}^{\circ}$ de observaciones

$\mathrm{R}^{2}$ ajustado

0,412

0,429

0,371

Fuente: elaboración propia sobre la base de datos de la evaluación internacional PISA 2009.

a Para cada área de evaluación fueron estimadas 405 regresiones mediante mínimos cuadrados ponderados.

Nota: desviación estándar entre paréntesis.

$\mathrm{t}$ Variable no significativa al nivel de un $5 \%$. 
Costa Rica: descomposición del efecto de cada factor de la FPE en el rendimiento escolar de los jóvenes de 15 años, 2009

(En porcentajes)

\begin{tabular}{lrrr}
\hline & Lectura & Matemáticas & Ciencias \\
\hline Factores familiares y del estudiante & 30,91 & 31,84 & 27,04 \\
$\quad$ Mujer & 0,87 & 2,11 \\
Asistió a preescolar & 0,74 & 0,39 \\
Año de colegio al que asiste & 20,90 & $1,36,61$ \\
Estatus ocupacional & 0,22 & 0,00 \\
Educación de la madre & 1,14 & 17,54 & 1,17 \\
Libros en el hogar & 2,71 & 0,19 & 2,64 \\
Posesiones y recursos educativos en el hogar & 2,41 & 2,39 & 2,41 \\
$\quad$ Efecto de pares (peer effect) & 1,92 & 1,88 & 1,71 \\
Factores escolares & 9,40 & 3,13 & 9,35 \\
Características del centro educativo & 8,92 & 1,33 & 8,92 \\
$\quad$ Colegio público & 4,11 & 10,23 & 6,04 \\
Colegio único en la zona & 0,03 & 9,55 & 0,01 \\
Directora & 0,16 & 3,31 & 0,00 \\
$\quad$ Indice de recursos educativos en el colegio & 4,45 & 0,00 & 3,42 \\
Computadoras disponibles para estudiar & 0,13 & 0,20 & 0,00 \\
Carencia de libros, laboratorio y computadoras & 0,04 & 4,22 & $-0,55$ \\
Características de los profesores & 0,48 & 0,19 & 0,43 \\
Factores institucionales & 0,89 & 1,63 & 0,71 \\
\hline Total & 41,2 & 0,68 & 37,1 \\
\hline
\end{tabular}

Fuente: elaboración propia sobre la base de datos de la evaluación internacional PISA 2009.

FPE: función de producción educativa. 
Costa Rica: descomposición del efecto de cada factor de la FPE en el rendimiento escolar de los jóvenes de 15 años, por sector educativo, 2009

(En porcentajes)

\begin{tabular}{|c|c|c|c|c|c|c|}
\hline & \multicolumn{2}{|c|}{ Lectura } & \multicolumn{2}{|c|}{ Matemáticas } & \multicolumn{2}{|c|}{ Ciencias } \\
\hline & Público & Privado & Público & Privado & Público & Privado \\
\hline Factores familiares y del estudiante & 27,41 & 34,71 & 29,96 & 29,02 & 24,58 & 29,83 \\
\hline Mujer & 0,87 & 0,05 & 4,84 & 8,94 & 2,11 & 7,43 \\
\hline Nacionalidad costarricense & 0,22 & 0,03 & 0,14 & 0,11 & 0,04 & 0,01 \\
\hline Asistió a preescolar & 0,61 & 4,75 & 0,81 & 1,41 & 0,31 & 0,05 \\
\hline Año de colegio al que asiste & 20,01 & 16,50 & 18,05 & 8,72 & 17,32 & 11,42 \\
\hline Estatus ocupacional & 0,33 & 0,27 & $-0,23$ & 0,26 & $-0,20$ & $-0,32$ \\
\hline Educación de la madre & 0,91 & 0,01 & 2,08 & 0,72 & 1,00 & 0,09 \\
\hline Libros en el hogar & 1,78 & 3,83 & 1,54 & 1,14 & 1,87 & 3,80 \\
\hline Posesiones y recursos educativos en el hogar & 2,56 & 6,86 & 2,49 & 4,79 & 1,99 & 4,98 \\
\hline Efecto de pares (peer effect) & 0,12 & 2,41 & 0,24 & 2,93 & 0,14 & 1,92 \\
\hline Factores escolares & 1,14 & 6,71 & 2,71 & 8,87 & 1,48 & 11,88 \\
\hline Características del centro educativo & 0,81 & 6,34 & 2,2 & 7,49 & 1,13 & 7,31 \\
\hline Colegio único en la zona & 0,02 & 0,03 & 0,01 & 0,10 & 0,00 & 1,95 \\
\hline Directora & 0,11 & 0,09 & 0,14 & $-0,17$ & 0,00 & 0,48 \\
\hline Índice de recursos educativos en el colegio & 0,31 & 0,01 & 1,36 & 0,00 & 1,02 & 0,23 \\
\hline Computadoras disponibles para estudiar & 0,29 & $-0,68$ & 0,06 & $-1,00$ & 0,04 & $-0,07$ \\
\hline Carencia de libros, laboratorio y computadoras & 0,08 & 6,89 & 0,63 & 8,56 & 0,07 & 4,72 \\
\hline Características de los profesores & 0,33 & 0,37 & 0,51 & 1,38 & 0,35 & 4,57 \\
\hline Factores institucionales & 1,23 & 13,02 & 1,98 & 14,64 & 1,55 & 9,28 \\
\hline Total & 29,78 & 54,44 & 34,65 & 52,53 & 27,61 & 50,99 \\
\hline
\end{tabular}

Fuente: cálculos propios sobre la base de datos de la evaluación internacional PISA 2009.

FPE: función de producción educativa. 
Card, D. y A. Krueger (1996), "School resources and student outcomes: an overview of the literature and new evidence from North and South Carolina", Journal of Economic Perspectives, vol. 10, $\mathrm{N}^{\circ}$ 4, Nashville, Tennessee, American Economic Association.

Coleman, J. (1966), Equality of Educational Opportunity, Washington, D.C., Departamento de Salud, Educación y Bienestar.

Contreras, D. y S. Gallegos (2011), "Desigualdad salarial en América Latina: Una década de cambios", Revista de la CEPAL, $\mathrm{N}^{\circ} 103$ (LC/G.2487-P), Santiago de Chile, abril.

Cristia, J., A. Czerwonko y P. Garofalo (2010), "Does ICT Increase Years of Education? Evidence from Peru", OVE Working Papers, $\mathrm{N}^{\circ} 110$, Washington, D.C., Banco Interamericano de Desarrollo.

Cristia, P. y otros (2012), "Technology and child development: evidence from the one laptop per child program", $I D B$ Working Paper Series, $\mathrm{N}^{\circ}$ 304, Washington, D.C., Department of Research and Chief Economist, Banco Interamericano de Desarrollo.

Díaz Porras, R. y R. Jiménez Rodríguez (1980), “Consideraciones económicas sobre el sistema educativo costarricense", tesis para optar al grado de licenciatura, San José, Facultad de Ciencias Económicas, Universidad de Costa Rica.

Epple, D. y R. Romano (1998), "Competition between private and public schools, vouchers, and peer-group effects", American Economic Review, vol. 88, $\mathrm{N}^{\circ}$ 1, Nashville, Tennessee, American Economic Association.

Estado de la Nación (2011), Informe III. Nuevos Instrumentos para el Análisis de la Educación en Costa Rica, San José, Programa Estado de La Nación.

Fields, G.S. (2003), "Accounting for income inequality and its change: a new method, with application to the distribution of earnings in the United States", Research in Labor Economics, vol. 22, Bingley, Emerald.

Formichella, M. (2011), " ¿Se debe el mayor rendimiento de las escuelas de gestión privada en la Argentina al tipo de administración?", Revista de la CEPAL, № 105 (LC/G.2508-P), Santiago de Chile.

Greenwald, R., L. Hedges y R. Laine (1996), "The effect of school resources on student achievement", Review of Educational Research, vol. 66, $\mathrm{N}^{\circ}$ 3, Washington, D.C., American Educational Research Association.

Hanushek, E. (1986), "The economics of schooling: production and efficiency in public schools", Journal of Economic Literature, vol. 24, $\mathrm{N}^{\circ} 3$, Nashville, Tennessee, American Economic Association.

(1979), "Conceptual and empirical issues in the estimation of educational production functions", The Journal of Human Resources, vol. 14, $\mathrm{N}^{\circ} 3$, Madison, University of Wisconsin Press.

Hanushek, E. y L. Woessmann (2010), "The Economics of International Differences in Educational Achievement", NBER Working Paper, $\mathrm{N}^{\circ}$ 15949, Cambridge, Massachusetts, National Bureau of Economic Research.

(2005), "Does Educational Tracking Affect Performance and Inequality? Differences-in-differences Evidence Across
Countries", NBER Working Paper, $\mathrm{N}^{\circ} 11124$, Cambridge, Massachusetts, National Bureau of Economic Research.

Heshmati, A. (2004), "A review of decomposition of income inequality", IZA Discussion Paper, $\mathrm{N}^{\circ} 1221$, Bonn, Institute for the Study of Labor (IZA).

Kremer, M. (1995), "Research on schooling: what we know and what we don't. A comment on Hanushek", World Bank Research Observer, vol. 10, $\mathrm{N}^{\circ}$ 2, Washington, D.C., Oxford University Press.

Lee, J. y R. Barro (2001), "Schooling quality in a cross-section of countries", Economica, vol. 68, $\mathrm{N}^{\circ} 272$, Londres, London School of Economics and Political Science.

MEP (Ministerio de Educación Pública) (2010), Educando en tiempos de cambio: Memoria institucional 2006-2010, San José.

Mizala, A. y P. Romaguera (2002), "Equity and educational performance", Documentos de trabajo, $\mathrm{N}^{\circ} 136$, Santiago de Chile, Centro de Economía Aplicada, Universidad de Chile.

Morduch, J. y T. Sicular (2002), "Rethinking inequality decomposition, with evidence from rural China", Economic Journal, vol. 112, $\mathrm{N}^{\circ} 476$, St. Andrews, Royal Economic Society.

Moreira Mora, T. (2009), "Factores endógenos y exógenos asociados al rendimiento en matemática: Un análisis multinivel”, Revista Educación, vol. 33, № 2, San José, Universidad de Costa Rica.

ocDE (Organización de Cooperación y Desarrollo Económicos) (2012), PISA 2009 Technical Report, París, OECD Publishing. (2009), PISA Data Analysis Manual: SPSS and SAS, París, OECD Publishing.

Shorrocks, A. (1984), "Inequality decomposition by population subgroups", Econometrica, vol. 52, N ${ }^{\circ} 6$, Nueva York, The Econometric Society.

(1982), "Inequality decomposition by factor components", Econometrica, vol. 50, $\mathrm{N}^{\circ} 1$, Nueva York, The Econometric Society.

(1980), "The class of additively decomposable inequality", Econometrica, vol. 48, $\mathrm{N}^{\circ} 3$, Nueva York, The Econometric Society.

Todd, P.E. y K.I. Wolpin (2003), "On the specification and estimation of the production function for cognitive achievement", The Economic Journal, vol. 113, N ${ }^{\circ} 485$, St. Andrews, Royal Economic Society.

UNESCO (Organización de las Naciones Unidas para la Educación, la Ciencia y la Cultura) (2008), Los aprendizajes de los estudiantes de América Latina y el Caribe, Santiago de Chile, Oficina Regional de Educación de la UNESCO para América Latina y el Caribe (OREALC/UNESCO).

Urzúa, S. y G. Veramendi (2011), "The impact of out-of-home child care centers on early childhood development", IDB Working Paper Series, $\mathrm{N}^{\circ}$ IDB-WP-240, Washington, D.C., Banco Interamericano de Desarrollo.

Vegas, E. y J. Petrow (2007), Raising Student Achievement in Latin America: The Challenge for the 21st Century, Washington, D.C., Banco Mundial.

Walker, M. (2011), PISA 2009 PLUS Results: Performance of 15-yearolds in Reading, Mathematics and Science for 10 Additional Participants, Melbourne, ACER Press.

Woessmann, L. (2011), Schooling Resources, Educational Institutions, and Student Performance: The International Evidence, Kiel, Kiel Institute for the World Economy. 